\title{
The role of plasma D-dimer concentration in the exclusion of pulmonary embolism
}

\author{
Edwin J. R. van Beek, ${ }^{1}$ B. Ed Schenk, ${ }^{2}$ Bowine C. Michel, ${ }^{3}$ Bram van den Ende, ${ }^{1,4}$ Dees P. M. Brandjes, ${ }^{1,2}$ \\ Yvonne T. van der Heide, ${ }^{4}$ Patrick M. M. Bossuyt ${ }^{5}$ and Harry R. Büller ${ }^{1}{ }^{1}$ Centre for Haemostasis, Thrombosis, \\ Atherosclerosis and Inflammation Research and ${ }^{5}$ Department of Clinical Epidemiology and Biostatistics, Academic Medical \\ Centre, Amsterdam, Departments of ${ }^{2}$ Internal Medicine and ${ }^{4}$ Clinical Chemistry, Slotervaart Ziekenhuis, Amsterdam, and \\ ${ }^{3}$ Institute for Medical Technology Assessment, Erasmus University, Rotterdam, The Netherlands
}

Received 26 June 1995; accepted for publication 20 October 1995

\begin{abstract}
Summary. Objective. To determine the role of four ELISA D-dimer assays in the exclusion of pulmonary embolism.

Design. Blinded comparison using pulmonary angiography and/or lung scintigraphy as a reference method.

Setting. A secondary and tertiary referral centre.

Patients and methods. Consecutive patients with suspected pulmonary embolism underwent lung scintigraphy, followed by angiography if a non-diagnostic result was obtained. Comorbid conditions resulting in elevated plasma D-dimer levels were defined a priori. Cut-off levels for $100 \%$ sensitivity were determined. A decision-analytic model was used to determine effectiveness and costs in the management pulmonary embolism.

Main outcome measures. The exclusion efficacy of the various assays at a sensitivity of $100 \%$, and cost-effectiveness.

Results. A total of 179 patients were included ( 78 inpatients and 101 outpatients; 74 patients had comorbid conditions). Pulmonary embolism could be adequately excluded in between
\end{abstract}

$8 \%$ and $18 \%$ of all patients, and in between $3 \%$ and $7 \%$ and $11 \%$ and $27 \%$ of inpatients and outpatients, respectively, depending on the assay used. D-dimer assays could exclude pulmonary embolism in $<5 \%$ of patients with comorbid conditions, whereas this increased to $14-32 \%$ in outpatients without comorbid conditions. A cost-effectiveness analysis showed a cost reduction of $10 \%$ at a specificity of $30 \%$, largely due to a $28 \%$ decrease in angiography requirements. Furthermore, for every $2 \%$ decrease in sensitivity, one per 1000 evaluated patientswould die as a result of inadequately treated pulmonary embolism.

Conclusion. D-dimer ELISA assays may have a role in the exclusion of pulmonary embolism in symptomatic outpatients, where the application may reduce angiography by $30 \%$ and costs by $10 \%$.

Keywords: pulmonary embolism, diagnosis, D-dimer, ELISA, cost-effectiveness.
Clinically suspected pulmonary embolism is a frequent and difficult diagnostic challenge. Several studies have shown that annually about three per 1000 people are suspected of this disorder (Anderson et al, 1991; van Beek et al, 1991), whereas only $30 \%$ will have the diagnosis confirmed (Hull et al, 1983; PIOPED, 1990; McBride et al, 1986). To establish or exclude pulmonary embolism often requires multiple, sometimes invasive, and costly diagnostic procedures. Although in recent years several major advances have been made, such as the clinical validity of a normal perfusion lung scan to refute pulmonary embolism or a high probability scan result as sufficient proof for the diagnosis to warrant longterm anticoagulant therapy, there remains controversy over the best strategy for the $50-60 \%$ of symptomatic patients Correspondence: Dr E. J. R. van Beek, F4-136, Academic Medical Centre, Meibergdreef 9, 1105 AZ Amsterdam, The Netherlands. who have neither of the above lung scan results. In these patients with lung scan results that are designated as nondiagnostic (Hull \& Raskob, 1991; Moser, 1990), several management strategies are currently proposed. They vary from pulmonary arteriography in all patients to the use of (in various combinations) clinical decision rules (Patil et al, 1993; Stein et al, 1993a), tests for the detection of deep venous thrombosis (Kruit et al, 1991; Stein et al, 1993b; Hull et al, 1994) or computed tomography (Remy-Jardin et al, 1992; Teigen et al, 1993).

Recently, several investigators have assessed the potential value of detecting certain markers of coagulation or fibrinolysis activation, based on the concept that increased levels in peripheral blood reflect the presence of thrombosis in the circulation. In particular, the utility of measuring the degradation product of cross-linked fibrin (D-dimer) in plasma 


\section{Edwin J. R. van Beek et al}

has been evaluated in patients with clinically suspected pulmonary embolism (Bounameaux et al, 1991; Leitha et al, 1991; Demers et al, 1992; Harrison et al, 1993; Goldhaber et al, 1993). From these studies it has become apparent that the majority of patients with pulmonary embolism have an elevated plasma D-dimer concentration. However, patients with clinical conditions mimicking pulmonary embolism often have similar concentrations. Therefore the most promising application of this test lies in the exclusion of pulmonary emboli in symptomatic patients, i.e. in those patients with normal plasma D-dimer concentrations (Harrison et al, 1993; Goldhaber et al, 1993; Ginsberg et al, 1993; van Beek et al, 1993; Bounameaux et al, 1994). Such an approach is only acceptable if virtually none of the patients with pulmonary embolism is missed, so that, in the future, anticoagulant treatment can be safely withheld in patients with symptoms suggesting pulmonary embolism and normal D-dimer test results.

With this high requirement of a sensitivity approaching $100 \%$ with a consequent negative predictive value of $100 \%$, it becomes important to assess how many patients of the initial cohort with clinically suspected pulmonary embolism can be safely identified and spared further investigation and treatment. Furthermore, it is unknown whether there is an advantage to using this test in all symptomatic patients or to limit the determination to certain subgroups, such as outpatients or those without diseases known to be associated with intravascular fibrin deposition. Finally, there is controversy whether the D-dimer test should be used early or later in the diagnostic work-up of patients suspected of having pulmonary emboli (Bounameaux et al, 1991; Harrison et al, 1993; Perrier et al, 1994; Quinn et al, 1994).

We therefore undertook a cross-sectional study in a consecutive series of patients with clinically suspected pulmonary embolism. First we estimated, for four different assays, the plasma D-dimer concentration which yielded a sensitivity of $100 \%$. Subsequently, we calculated the percentage of patients from the entire cohort in whom D-dimer was able to exclude pulmonary emboli in all patients and in certain subgroups: newly seen or admitted patients (outpatients), previously admitted patients (inpatients) and patients with or without comorbid conditions. Finally, using a decision-analytic model, the best time to perform D-dimer tests in order to obtain the highest diagnostic utility was determined.

\section{MATERIALS AND METHODS}

Patients. Consecutive patients (both in- and outpatients), who underwent diagnostic work-up for clinically suspected pulmonary embolism in two large teaching hospitals in Amsterdam, The Netherlands, were eligible for inclusion into the study. Clinical and demographic characteristics were prospectively collected. All patients underwent lung scintigraphy. Perfusion lung scintigraphy was performed using ${ }^{99} \mathrm{~m}_{\text {Technetium-labelled macro-aggregates of albumin (Sorin }}$ Biomedica, Vercelli, Italy), while ventilation images were obtained using ${ }^{81 \mathrm{~m}}$ Krypton gas (Cygne by, Eindhoven, The Netherlands). Lung scans were classified as normal, high probability for pulmonary embolism (defined as one or more segmental perfusion defects with normal ventilation), or nonhigh probability (non-diagnostic) (PIOPED, 1990; Hull et al, 1985; Lensing et al, 1992).

All patients with non-diagnostic lung scan results underwent pulmonary angiography. Selective intra-arterial pulmonary angiography was performed using low-osmolar contrast media. Angiography was considered to demonstrate pulmonary emboli if a filling defect or an acute cut-off of an arterial branch with a diameter of $2 \cdot 5 \mathrm{~mm}$ or more was seen in two projections (PIOPED, 1990; Dalen et al, 1971).

Patients were classified as having pulmonary embolism if a high probability lung scan result was obtained or if pulmonary emboliwere shownon pulmonary angiography. Pulmonary embolism was excluded if a normal lung scan or angiogram was obtained and 6 months follow-up was uneventful with respect to symptomatic venous thrombo-embolism.

The study was approved by the respective Institutional Review Boards and written informed consent was obtained from all patients.

Plasma samples. Prior to heparinization, blood was collected from each patient. Platelet-poor plasma was obtained by centrifuging $9 \mathrm{ml}$ of venous blood collected into $1 \mathrm{ml}$ of $0 \cdot 105 \mathrm{~m}$ sodium citrate solution (Becton Dickinson, New Jersey, U.S.A.) for $20 \mathrm{~min}$ at $3000 \mathrm{rpm}(1200 \mathrm{~g})$ at $15^{\circ} \mathrm{C}$. All samples were stored at $-70^{\circ} \mathrm{C}$. Assays were performed within 6 months of blood collection. All samples were analysed in duplicate, without knowledge of the results of other diagnostic tests that were performed.

Four EIA plasma D-dimer assays were evaluated: Dimertest I (Agen Test Technologies, Acacia Ridge, Qld, Australia), Enzygnost D-dimer (Behringwerke, Marburg, Germany), Fibrinostika FbDP (Organon Teknika, Boxtel, The Netherlands) and Asserachrom D-di (Diagnostica Stago, Asnières, France). The discriminant values used for raised plasma D-dimer levels were $50 \mu \mathrm{g} / \mathrm{l}, 40 \mu \mathrm{g} / \mathrm{l}, 550 \mu \mathrm{g} / \mathrm{l}$ and $400 \mu \mathrm{g} / \mathrm{l}$, respectively.

Study design. Since the basis of the clinical utility of D-dimer tests rests with the safe exclusion of pulmonary embolism, we first determined, in all patients, the cut-off value for each assay. In order to have optimal confidence that a normal D-dimer would exclude pulmonary embolism, the D-dimer plasma concentration corresponding to the lowest value observed in patients with proven pulmonary embolism was determined. By definition, this leads to a sensitivity of $100 \%$. Subsequently, we calculated the exclusion efficacy as the percentage of patients of the entire group in whom the diagnosis would have been adequately excluded by the finding of normal plasma D-dimer concentration.

Since the presence of other disorders may increase plasma D-dimer levels, subgroup analyses were performed to evaluate the influence of comorbid conditions and of prior admission (i.e. the development of symptoms in outpatients or in patients already admitted for other reasons). The comorbid conditions were identified a priori according to previously published definitions (Demers et al, 1992). Briefly, patients were classified as having comorbid conditions if the following circumstances or disorders were present: $<10 \mathrm{~d}$ following either surgery, trauma, myocardial infarction or cerebrovas- 
cular accident; septicaemia, diffuse intravascular coagulation or acute infection; pregnancy or $<10 \mathrm{~d}$ postpartum; active collagen vascular disease; or metastatic cancer.

Finally, with the use of a decision-analytic model, we attempted to define the optimal place of the plasma D-dimer assay in the diagnostic management of patients with clinically suspected pulmonary embolism. Based on previously reported analyses, we selected two strategies which are widely used and were previously shown to be costeffective (PIOPED, 1990; Oudkerk et al, 1993; Hull et al, 1994). The analysis for each of these two strategies was expanded with the inclusion of D-dimer as a first test or as an additional test only in patients with non-diagnostic lung scan results, resulting in two groups of strategies.

The main assumptions in this model are related to complications of invasive diagnostic methods, complications of anticoagulant therapy and the natural history of pulmonary embolism (Oudkerk et al, 1993). From several reports in the literature, the morbidity and mortality related to pulmonary angiography was estimated to be $1.9 \%$ and $0 \cdot 2 \%$, respectively (Dalen et al, 1971; Mills et al, 1980; Stein et al, 1992). The risk of major bleeding due to anticoagulant therapy was estimated at $1 \%$, whereas fatal haemorrhage was thought to occur in $0 \cdot 25 \%$ of patients, for a 3 -month course of anticoagulants (Hull et al, 1982; Levine et al, 1992; van der Meer et al, 1993). The natural history of untreated pulmonary embolism can only be properly derived from one study, in which non-fatal recurrence was observed in $26 \%$ and fatal recurrence in $26 \%$ of untreated patients (Barritt \& Jordan, 1960). For further details of these and other assumptions the reader is referred to the full paper (Oudkerk et al, 1993).

Group I comprised [1] perfusion-ventilation lung scintigraphy with ultrasonography of the deep leg veins if the lung scan result was non-diagnostic and pulmonary angiography if ultrasonography was normal; patients were treated if the lung scan was high probability, if ultrasonography was abnormal or if angiography showed pulmonary emboli; [2] D-dimer as a first test, followed by strategy [1] if the D-dimer level was above the cut-off level; [3] perfusion-ventilation lung scintigraphy with D-dimer if the lung scan result was nondiagnostic, ultrasonography of the leg if the D-dimer was above the cut-off level, and pulmonary angiography if ultrasonography was normal; treatment as in the first strategy.

Group II contained the following strategies: [4] perfusionventilation lung scintigraphy with pulmonary angiography if the lung scan result was non-diagnostic; patients were treated if the lung scan was high probability or if angiography shows pulmonary emboli; [5] D-dimer as a first test, followed by strategy [4] if the D-dimer level was above the cut-off level. Finally, the sixth strategy includes perfusion-ventilation lung scintigraphy with D-dimer if the lung scan result was non-diagnostic, and angiography if the plasma D-dimer

Table I. Clinical and demographic characteristics of 179 patients with clinically suspected pulmonary embolism.

\begin{tabular}{lcc}
\hline & Inpatients $(n=78)$ & Outpatients $(n=101)$ \\
\hline Male/female & $40 / 38$ & $36 / 65$ \\
Mean age (range) (years) & $58 \cdot 5(22-92)$ & $56 \cdot 5(18-82)$ \\
Scintigraphic and angiographic findings & & \\
Normal perfusion lung scan & $21(27 \%)$ & $40(40 \%)$ \\
Normal angiogram & $19(24 \%)$ & $26(26 \%)$ \\
High-probability lung scan & $33(43 \%)$ & $25(24 \%)$ \\
Abnormal angiogram & $5(6 \%)$ & $10(10 \%)$ \\
Referring speciality/service & & \\
Medicine (including pulmonology) & $48(61 \%)$ & $47(47 \%)$ \\
Emergency department & $0(0 \%)$ & $41(41 \%)$ \\
Surgery & $16(21 \%)$ & $6(5 \%)$ \\
Other & $14(18 \%)$ & $7(7 \%)$ \\
Risk factors for venous thromboembolism & & $5(5 \%)$ \\
Recent surgery and/or trauma* & $13(17 \%)$ & $9(9 \%)$ \\
History of venous thromboembolism & $7(9 \%)$ & $3(3 \%)$ \\
Paralysis or paresis of lower limb(s) & $10(13 \%)$ & $0(0 \%)$ \\
Recent cerebral or myocardial infarction* & $3(4 \%)$ & \\
Coexisting diseases & & $20(20 \%)$ \\
Chronic obstructive pulmonary disease & $18(23 \%)$ & $3(11 \%)$ \\
Malignant disease & $18(23 \%)$ & $3(3 \%)$ \\
Acute infection & $13(17 \%)$ & \\
Active collagen vascular disease & $4(5 \%)$ & \\
\hline
\end{tabular}

* Recent is defined as within $10 \mathrm{~d}$ for inclusion into the study. 


\section{Edwin J. R. van Beek et al}

concentration was above the cut-off level; therapy as in strategy [4].

For comparison, we included an imaginary strategy which assumes correct diagnosis and therapy in all patients without diagnostic tests, the so-called clairvoyant strategy.

Data analysis. The McNemar $\chi^{2}$-test was used to compare differences between the defined subgroups. A $P$ value of $<0.05$ was regarded as statistically significant.

The cost-effectiveness analysis was performed using previously presented assumptions (Oudkerk et al, 1993). These assumptions consisted of prevalence of pulmonary embolism and deep venous thrombosis, sensitivity and specificity of diagnostic tests (angiography, lung scintigraphy and ultrasonography of the leg veins), morbidity and mortality of angiography and anticoagulant therapy, morbidity and mortality in the natural history of pulmonary embolism, and costs of procedures and therapy. Additionally, we assumed that the D-dimer assay was available $24 \mathrm{~h}$ per day and costs 40 ECU (or $35 \mathrm{UK} f$ ) per patient. The sensitivity of the D-dimer assay was set at $98 \%$, and a specificity of $30 \%$ was used for base-line analysis. The efficacy of the various strategies was assessed in terms of mortality, morbidity, costs, and percentage of patients requiring pulmonary angiography.

A sensitivity analysis was performed, which varied one parameter each time. The tested parameters (and the range evaluated) were: specificity of D-dimer assay (10-50\%), sensitivity of D-dimer assay (90-100\%), prevalence of pulmonary embolism $(20-50 \%)$, risk of major haemorrhage $(0 \cdot 5-5 \%)$, sensitivity $(96-100 \%)$ and specificity $(92-100 \%)$ of pulmonary angiography.

\section{RESULTS}

\section{Study subjects}

A total of 203 consecutive patients with clinically suspected pulmonary embolism were entered into the study. In 24 $(12 \%)$ patients with a non-diagnostic lung scan result, angiography could not be performed due to existing contraindications (12 manifest heart failure, two myocarditis, three severe pulmonary hypertension, three severe dyspnoea with inability to lie flat, two thrombocytopenia $<20 \times 10^{9} / 1$, and two renal failure). Since no definite diagnosis could be obtained in these patients, they were excluded from the analysis. Hence, 179 patients, of whom 78 were inpatients and 101 were outpatients, were evaluated.

The clinical, demographic characteristics and scintigraphic and angiographic findings of the studied patients are listed in Table I. The mean age of the study population was 56.5 years; almost three-quarters of the patients had no known risk factors for thromboembolism, whereas malignant disease was present in $16 \%$. The mean delay between onset of symptoms and presentation was $2 \mathrm{~d}$ (range 0-60 d). Pulmonary embolism was excluded in 106 patients (61 normal perfusion lung scan and 45 normal pulmonary angiogram), whereas emboli were demonstrable in the remaining 73 patients ( 58 high probability lung scan and 15 positive angiogram), for a prevalence of pulmonary embolism of $41 \%$.
Table II. Percentage of patients with clinically suspected pulmonary embolism in whom this disease would have been adequately excluded by a normal D-dimer ELISA assay.

\begin{tabular}{lrc}
\hline & \multicolumn{2}{c}{$\begin{array}{c}\text { Percentage of patients } \\
\text { adequately excluded } \\
\text { by D-dimer }\end{array}$} \\
\cline { 2 - 3 } & $n$ & Range \\
\hline All patients & 179 & $8-18 \%$ \\
Inpatients & 78 & $3-6 \%$ \\
Outpatients & 101 & $11-27 \%$ \\
Presence of comorbidity & 74 & $0-5 \%$ \\
Absence of comorbidity & 105 & $13-27 \%$ \\
Outpatients without comorbidity & 76 & $14-32 \%$ \\
& & \\
\hline
\end{tabular}

*For definition of comorbid conditions see Materials and Methods section.

\section{Exclusion of pulmonary embolism}

The discriminant plasma concentrations of the four D-dimer assays were applied, and their value for the adequate exclusion of pulmonary embolism in all patients, and separately for in- and outpatients, presenting with clinical suspicion of this disorder, was determined. These results are described in Table II. The percentage of patients which could be excluded varied from $8 \%$ to $18 \%$ in all patients. This increased to up to $27 \%$ if only outpatients were assessed. All methods performed equally badly in inpatients.

When patients were grouped according to the presence or absence of comorbid conditions which may independently raise plasma D-dimer levels, the exclusion rate of all assays in patients with comorbidity was insufficient to be clinically useful (Table II). For all assays the exclusion rate was significantly better (varying from $13 \%$ to $27 \%$ ) in patients without comorbid conditions, and this improved only marginally if solely outpatients without comorbid conditions were analysed.

\section{Cost-effectiveness analysis}

The efficacy (in terms of mortality and morbidity rates), the estimated average cost per patient for diagnosis and treatment and the angiography rate required in the various management strategies for the entire study population of patients with clinically suspected pulmonary embolism, are shown in Table III. For reference, a theoretical (clairvoyant) strategy is given. The first three strategies all contain ultrasonography, but this test is omitted in the last three strategies. Addition of the D-dimer assay to the diagnostic strategies does not result in any significant change in the mortality rates, whereas morbidity decreases to a slightly lower level. Furthermore, regardless of the strategy employed, there is an approximate $10 \%$ cost-reduction in addition to a $25-28 \%$ reduction in the number of patients requiring pulmonary angiography. The timing for performing the D-dimer assay, either as an initial screening test or later in the diagnostic process, does not have major effects on the parameters of the cost-effectiveness analysis. 
Table III. Efficacy in terms of mortality and morbidity, costs and percentage of patients requiring pulmonary angiography in various diagnostictherapeutic strategies.

\begin{tabular}{|c|c|c|c|c|}
\hline Strategy & $\begin{array}{l}\text { Mortality } \\
\text { rate }(\%)\end{array}$ & $\begin{array}{l}\text { Morbidity } \\
\text { rate (\%) }\end{array}$ & $\begin{array}{l}\text { Costs/patient } \\
\left(\mathrm{ECU}^{*}\right)\end{array}$ & $\begin{array}{l}\text { Angiography } \\
\text { required (\%) }\end{array}$ \\
\hline Correct in all (clairvoyant) & $0 \cdot 69$ & $0 \cdot 92$ & 652 & 0 \\
\hline $\begin{array}{l}\text { 1. Perfusion-ventilation scan with ultrasonography } \\
\text { if scan shows non-high probability and angiography } \\
\text { if ultrasound is normal; treatment if scan shows high } \\
\text { probability, if ultrasound is abnormal, or if } \\
\text { angiography shows pulmonary embolism }\end{array}$ & $0 \cdot 87$ & $2 \cdot 17$ & 1265 & $61 \cdot 8$ \\
\hline 2. D-dimer followed by strategy 1 if D-dimer elevated & $0 \cdot 97$ & 1.97 & 1131 & $44 \cdot 4$ \\
\hline \multicolumn{5}{|l|}{$\begin{array}{l}\text { 3. Perfusion-ventilation scan with D-dimer if scan shows } \\
\text { non-high probability, ultrasonography if D-dimer } \\
\text { elevated and angiography if ultrasound is normal; } \\
\text { treatment as strategy } 1\end{array}$} \\
\hline $\begin{array}{l}\text { 4. Perfusion-ventilation scan with angiography if scan } \\
\text { shows non-high probability; treatment if scan shows } \\
\text { high probability or if angiogram shows pulmonary } \\
\text { embolism }\end{array}$ & $0 \cdot 94$ & $2 \cdot 41$ & 1254 & $72 \cdot 2$ \\
\hline 5. D-dimer followed by strategy 4 if D-dimer elevated & $1 \cdot 04$ & $2 \cdot 19$ & 1133 & $54 \cdot 4$ \\
\hline $\begin{array}{l}\text { 6. Perfusion-ventilation scan with D-dimer if scan shows } \\
\text { non-high probability and angiography if D-dimer } \\
\text { elevated; treatment as strategy } 4\end{array}$ & $0 \cdot 98$ & $2 \cdot 14$ & 1171 & $54 \cdot 4$ \\
\hline
\end{tabular}

ECU $=$ European Currency Unit.

Table IV. Sensitivity analysis and effects on mortality and costs of strategy using perfusion-ventilation lung scintigraphy, plasma D-dimer test, ultrasonography and angiography.*

\begin{tabular}{|c|c|c|c|c|c|}
\hline Variable & Base-line & Range & Effects on mortality & Effects on costs & Effects on angiography rate \\
\hline Specificity dimer & $30 \%$ & $50-10 \%$ & $\begin{array}{l}\text { Increase with decreasing } \\
\text { specificity }(0 \cdot 90-0 \cdot 94 \%)\end{array}$ & $\begin{array}{l}\text { Less costs-savings } \\
\text { with decreasing specificity } \\
\text { (13-1\% costs-savings) }\end{array}$ & $\begin{array}{l}\text { Less reduction } \\
\text { with decreasing specificity } \\
\text { (43-9\% reduction) }\end{array}$ \\
\hline Sensitivity D-dimer & $98 \%$ & $100-90 \%$ & $\begin{array}{l}\text { Increase } 0 \cdot 085 \% \text { with every } \\
2 \% \text { decrease in sensitivity } \\
(0 \cdot 83-1 \cdot 25 \%)\end{array}$ & $\begin{array}{l}\text { Increasing costs-savings } \\
\text { with decreasing sensitivity } \\
\text { ( } 7-10 \% \text { costs-savings) }\end{array}$ & $\begin{array}{l}\text { Virtually unchanged } \\
\text { (29\% reduction) }\end{array}$ \\
\hline Prevalence & $30 \%$ & $20-50 \%$ & $\begin{array}{l}\text { Increase }(0 \cdot 66-1 \cdot 44 \%) \\
\text { with increasing prevalence }\end{array}$ & $\begin{array}{l}\text { Less costs-savings } \\
\text { with increasing prevalence } \\
\text { (10-3\% costs-saving) }\end{array}$ & $\begin{array}{l}\text { Decreasing reduction } \\
\text { with increasing prevalence } \\
\text { ( } 37-24 \% \text { reduction) }\end{array}$ \\
\hline $\begin{array}{l}\text { Sensitivity } \\
\text { angiography }\end{array}$ & $98 \%$ & $90-100 \%$ & $\begin{array}{l}\text { Decrease }(1 \cdot 06-0 \cdot 88 \%) \\
\text { with increasing sensitivity }\end{array}$ & $\begin{array}{l}\text { Unchanged } \\
\text { (8\% costs-savings) }\end{array}$ & Not calculated \\
\hline $\begin{array}{l}\text { Specificity } \\
\text { angiography }\end{array}$ & $96 \%$ & $90-100 \%$ & Unchanged $(0 \cdot 92 \%)$ & $\begin{array}{l}\text { Less costs-savings (9-7\%) } \\
\text { with increasing specificity }\end{array}$ & Not calculated \\
\hline $\begin{array}{l}\text { Haemorrhagic } \\
\text { complications }\end{array}$ & $1 \cdot 25 \%$ & $0 \cdot 5-5 \%$ & $\begin{array}{l}\text { Increase }(0 \cdot 84-1 \cdot 16 \%) \\
\text { with increasing incidence }\end{array}$ & Not calculated & Not calculated \\
\hline
\end{tabular}

* Base-line values for strategy without D-dimer test: mortality $0 \cdot 87 \%$, costs $1265 \mathrm{ECU}$, angiography rate $61 \cdot 8 \%$.

${ }^{\dagger}$ Morbidity always less in strategy employing D-dimer than in strategy without D-dimer. 


\section{Edwin J. R. van Beek et al}

\section{Sensitivity analysis}

The results of the sensitivity analysis, which varied each of our assumptions, are described in Table IV. With decreasing specificity for the D-dimer test, mortality is virtually unaffected, whereas costs-savings are rapidly decreasing and the angiography rate returns to the $60-70 \%$ required in strategies which do not apply D-dimer (Table III). The influence of the sensitivity of the D-dimer assay on mortality is high: for every $2 \%$ reduction in sensitivity, mortality increases by approximately one per 1000 investigated patients, but extra costs-savings are marginal and the angiography rate is not further reduced as compared to the angiography rate with a D-dimer sensitivity of $100 \%$. Finally, changes in the prevalence of pulmonary embolism do not substantially affect the outcomes of the present analysis.

The sensitivity and specificity of pulmonary angiography have little influence on the outcome of the analysis. However, increasing haemorrhagic complications result in an increasing mortality of $0.7 \%$ for every $1 \%$ increase in bleeding complications.

\section{DISCUSSION}

The use of blood tests, such as plasma D-dimer methods, in the diagnosis of pulmonary embolism may have several advantages, such as its non-invasive nature, relatively low cost, and ease of use. Ideally, the proper evaluation of any diagnostic test, including the D-dimer assay, should involve three consecutive steps (Sackett et al, 1985; Büller et al, 1991). First, the technical aspects of the various D-dimer assays, such as intra- and inter-assay variability and the definition of normal and abnormal values, should be determined. The second step involves testing the accuracy of the D-dimer assay in a blind fashion in consecutive patients with or without a specific disease. Finally, clinical utility and validity should be ascertained.

We previously addressed the first step, and showed that the more accurate but laborious ELISA plasma D-dimer methods are preferred (van Beek et al, 1993). The results of the present investigation clearly demonstrate that D-dimer assays should only be used in excluding pulmonary embolism. Furthermore, it is shown that the highest clinical utility of this assay lies in excluding outpatients, where it may be used either as a screening test or following perfusion-ventilation lung scintigraphy.

It should be noted that, in order to make the D-dimer assay a reliable tool in the diagnostic work-up of patients with clinically suspected pulmonary embolism, one must use a cutoff value which results in virtually no patients with pulmonary embolism going unnoticed; hence, a sensitivity and negative predictive value approaching $100 \%$. Acceptance of sensitivity $<100 \%$ (Harrison et al, 1993; Goldhaber et al, 1993) may have serious adverse effects, as shown in our sensitivity analysis. It was calculated that one per 1000 evaluated patients with clinically suspected pulmonary embolism would die for every $2 \%$ decrease in sensitivity. Hence, in the present phase of evaluation of D-dimer assays standards need to be set high, because in subsequent application in clinical practice accuracy indices tend to be slightly lower.
Our analysis indicates that the D-dimer method was virtually unable to exclude pulmonary embolism in inpatients. This could in part be explained by the fact that pulmonary embolism was diagnosed in 35\% of outpatients and in $49 \%$ of inpatients, resulting in relatively fewer patients with possible normal D-dimer values in inpatients. Furthermore, comorbid conditions were present in $87 \%$ of inpatients and only $25 \%$ of outpatients $(P<0.001)$, which further reduced the possibility of obtaining a normal D-dimer value in inpatients. These findings of the effects of comorbid conditions and prior admission are in agreement with recent Canadian and Swiss studies (Demers et al, 1992; Raimondi et al, 1993).

The study population consisted of consecutive in- and outpatients with symptoms suggestive for pulmonary embolism and prevalence of proven pulmonary emboli of approximately $40 \%$. When taking into account that lung scan criteria may vary, the distribution of the lung scan findings in normal, high probability and non-high probability categories is in accordance with earlier studies (Hull et al, 1983; PIOPED, 1990; Goldhaber et al, 1993). Therefore we believe that our findings are relevant for all patients with symptomatic pulmonary embolism seen in a teaching hospital.

The place of D-dimer assays in a diagnostic strategy is currently debated (Bounameaux et al, 1991; Harrison et al, 1993; Perrier et al, 1994; Quinn et al, 1994). In our decisionanalytic model there did not appear to be a substantial difference between using D-dimer assays as an initial screening test or after an abnormal lung scan result has been obtained. In both applications of the D-dimer test a moderate cost-reduction of $10 \%$ was observed. This is largely the result of a reduced requirement to perform angiography and depends significantly on the specificity of the ELISA method used. If the specificity of the assay decreases below 20-25\% this beneficial effect on the angiography rate and costs disappears. The observed difference between the various ELISA methods illustrates the risk of extrapolating the findings with one method to other assays.

Although not analysed in this study, the time required to perform the D-dimer assay may be another factor in deciding the optimal place. When performed as a first test, a time delay for further testing of several hours may be incurred. On the other hand, when the test is performed later, this delay is less likely to be important. In clinical practice virtually all symptomatic patients will be treated with anticoagulants pending a definitive diagnosis. Since the outcome of using D-dimer as an initial test is similar to its use following lung scintigraphy, it is probably best to use the test in patients with abnormal lung scan findings.

The institution of heparin therapy could influence plasma D-dimer levels. A previous study showed that minimal changes may be expected to occur in the first few days of heparin therapy (Estivals et al, 1991). It appears that these changes will not have a significant effect within the first $24 \mathrm{~h}$. However, since more definitive data on this important issue are lacking, the plasma samples should preferably be obtained before heparinization takes place.

The cut-off values for a sensitivity of $100 \%$ clearly determine the specificity and consequently the number of patients in whom pulmonary embolism could be adequately 
excluded. One could argue that one patient could thus negatively influence the results in our series. However, the cut-off values never relied on a single low value. In patients with proven pulmonary embolism, several patients had lower D-dimer plasma concentrations which led to the determination of the appropriate cut-off values.

Finally, the present decision-analytic model does not take the prior probability of disease into account. Several reports have suggested that the use of this information (in combination with the D-dimer test result) may further improve clinical management (Stein et al, 1993a; Patil et al, 1993; Perrier et al, 1994).

In conclusion, the observations in this study suggest that the use of studied D-dimer assays in patients with clinically suspected pulmonary embolism should be limited to outpatients, where the application appears cost-effective. Moreover, it appears that the tests should be used following lung scintigraphy. We believe that a sensitivity approaching 100\% must be maintained in order to guarantee safe practice in the adequate exclusion of pulmonary embolism. This practice, however, needs to be confirmed in carefully designed management studies.

\section{ACKNOWLEDGMENTS}

Dr Büller is the recipient of a fellowship from the Royal Netherlands Academy of Arts and Sciences. The study was supported by a grant from the Netherlands Health Executive Insurance Board (OG91-036).

We are grateful for the technical assistance by all members of the Research Coagulation Laboratory at the Academic Medical Centre and the Laboratory for Clinical Chemistry at Slotervaart Hospital.

We thank Agen Test Laboratories, Boehringer Mannheim/ Stago, Hoechst/Behring and Organon Teknika for supplying the D-dimer reagents. The design and conduct of the study, and data analysis and manuscript preparation, remained independent of these sponsors.

\section{REFERENCES}

Anderson, F.A., Wheeler, H.B., Goldberg, R.J., Hosmer, D.W., Patwardhan, N.A., Jovanovic, B., Forcier, A. \& Dalen, J.E. (1991) A population-based perspective of the hospital incidence and casefatality rates of deep vein thrombosis and pulmonary embolism: the Worcester DVT study. Archives of Internal Medicine, 151, 933-938.

Barritt, D.W. \& Jordan, S.C. (1960) Anticoagulant drugs in the treatment of pulmonary embolism: a controlled study. Lancet, i, 1309-1312.

Bounameaux, H., Cirafici, P., de Moerloose, P., Schneider, P.A., Slosman, D., Reber, G. \& Unger, P.F. (1991) Measurement of D-dimer in plasma as diagnostic aid in suspected pulmonary embolism. Lancet, 337, 196-200.

Bounameaux, H., de Moerloose, P., Perrier, A. \& Reber, G. (1994) Plasma measurement of D-dimer as diagnostic aid in suspected venous thromboembolism: an overview. Thrombosis and Haemostasis, 71, 1-6.

Büller, H.R., Lensing, A.W.A., Hirsh, J. \& ten Cate, J.W. (1991) Deep vein thrombosis: new non-invasive diagnostic tests. Thrombosis and Haemostasis, 66, 133-137.
Dalen, J.E., Brooks, H.L., Johnson, L.W., Meister, S.G., Szucs, M.M. \& Dexter, L. (1971) Pulmonary angiography in acute pulmonary embolism: indications, techniques, and results in 367 patients. American Heart Journal, 81, 175-185.

Demers, C., Ginsberg, J.S., Johnston, M., Brill-Edwards, P. \& Panju, A. (1992) D-dimer and thrombin-antithrombin III complexes in patients with clinically suspected pulmonary embolism. Thrombosis and Haemostasis, 67, 408-412.

Estivals, M., Pelzer, H., Sie, P., Pichon, J., Boccalon, H. \& Boneu, B. (1991) Prothrombin fragment $1+2$, thrombin-antithrombin III complexes and D-dimers in acute deep vein thrombosis: effects of heparin treatment. British Journal of Haematology, 78, 421-424.

Ginsberg, J.S., Brill-Edwards, P.A., Demers, C., Donovan, D. \& Panju, A. (1993) D-dimer in patients with clinically suspected pulmonary embolism. Chest, 104, 1679-1684.

Goldhaber, S.Z., Simons, G.R., Elliott, C.G., Haire, W.D., Toltins, R., Blacklow, S.C., Doolittle, M.H. \& Weinberg, D.S. (1993) Qualitative plasma D-dimer levels among patients undergoing pulmonary angiography for suspected pulmonary embolism. Journal of the American Medical Association, 270, 2819-2822.

Harrison, K.A., Haire, W.D., Pappas, A.A., Purnell, G., Palmer, S., Holdeman, K.P., Fink, L.M. \& Dalrymple, G.V. (1993) Plasma Ddimer: a useful tool for evaluating suspected pulmonary embolus. Journal of Nuclear Medicine, 34, 896-898.

Hull, R.D., Hirsh, J., Carter, C.J., Jay, R.M., Dodd, P.E., Ockelford, P.A., Coates, G., Gill, G.J., Turpie, A.G., Doyle, D.J., Buller, H.R. \& Raskob, G.E. (1993) Pulmonary angiography, ventilation lung scanning, and venography for clinically suspected pulmonary embolism with abnormal perfusion lung scan. Annals of Internal Medicine, 98, 891-899.

Hull, R.D., Hirsh, J., Carter, C.J., Raskog, G.E., Gill, G.J., Jay, R.M., Leclerc, J.R., David, M. \& Coates, G. (1995) Diagnostic value of ventilation-perfusion lung scanning in patients with suspected pulmonary embolism. Chest, 88, 819-828.

Hull, R.D., Hirsh, J., Jay, R., Carter, C.J., England, C., Gent, M., Turpie, A.G.G., McLoughlin, D., Dodd, P., Thomas, M., Raskob, G. \& Ockelford, P. (1982) Different intensities of oral anticoagulant therapy in the treatment of proximal vein thrombosis. New England Journal of Medicine, 307, 1676-1681.

Hull, R.D. \& Raskob, G.E. (1991) Low-probability lung scan findings: a need for change. Annals of Internal Medicine, 114, 142-143.

Hull, R.D., Raskob, G.E., Ginsberg, J.S., Panju, A.A., Brill-Edwards, P., Coates, G. \& Pineo, G.F. (1994) A non-invasive strategy for the treatment of patients with suspected pulmonary embolism. Archives of Internal Mediciene, 154, 289-297.

Kruit, W.H.J., de Boer, A.C., Sing, A.K. \& van Roon, F. (1991) The significance of venography in the management of patients with clinically suspected pulmonary embolism. Journal of Internal Medicine, 230, 3333-339.

Leitha, T., Speiser, W. \& Dudczak, R. (1991) Efficacy of D-dimer and thrombin-antithrombin III complexes determination as screening tests before lung scanning. Chest, 100, 1536-1541.

Lensing, A.W.A., van Beek, E..J.R., Demers, C., Tiel-van Buul, M.M.C., Yakemchuk, V., van Dongen, A., Coates, G., Ginsberg, J.S., Hirsh, J., ten Cate, J.W. \& Büller, H.R. (1992) Ventilation-perfusion scanning and the diagnosis of pulmonary embolism: improvement of observer agreement by the use of a lung segment reference chart. Thrombosis and Haemostasis, 68, 245-249.

Levine, M.N., Hirsh, J., Landefeld, S. \& Raskob, G. (1992) Hemorrhagic complications of anticoagulant treatment. Chest, 102, 352S-363S.

McBride, K., LaMorte, W.W. \& Menzoian, J.O. (1986) Can ventilationperfusion scans accurately diagnose acute pulmonary embolism? Archives of Surgery, 121, 754-757.

Mills, S.R., Jackson, D.C., Older, R.A., Heaston, D.K. \& Moore, A.V. 


\section{Edwin J. R. van Beek et al}

(1980) The incidence, etiologies, and avoidance of complications of pulmonary angiography in a large series. Radiology, 136, 295299.

Moser, K.M. (1990) Venous thromboembolism. American Review of Respiratory Diseases, 141, 235-249.

Oudkerk, M., van Beek, E.J.R., van Putten, W.L.J. \& Büller, H.R. (1993) Cost-effectiveness analysis of various strategies in the diagnostic management of pulmonary embolism. Archives of Internal Medicine, 153, 947-954.

Patil, S., Henry, J.W., Rubenfire, M. \& Stein, P.D. (1993) Neural network in the clinical diagnosis of acute pulmonary embolism. Chest, 104, 1685-1689.

Perrier, A., Bounameaux, H., Morabia, A., deMoerloose, P., Slosman, D., Unger, P.F. \& Junod, A. (1994) Contribution of D-dimer plasma measurement and lower-limb venous ultrasound to the diagnosis of pulmonary embolism: a decision analysis model. American Heart Journal, 127, 624-635.

PIOPED Investigators (1990) Value of the ventilation/perfusion scan in acute pulmonary embolism: results of the prospective investigation of pulmonary embolism diagnosis (PIOPED). Journal of the American Medical Association, 263, 2753-2759.

Quinn, R.J., Nour, R., Butler, S.P., Glenn, D.W., Travers, P.L., Wellings, G. \& Kwan, Y.L. (1994) Pulmonary embolism in patients with intermediate probability lung scans: diagnosis with doppler venous US and D-dimer measurement. Radiology, 190, 509-511.

Raimondi, P., Bongard, O., de Moerloose, P., Reber, G., Waldvogel, F. \& Bounameaux, H. (1993) D-dimer plasma concentration in various clinical conditions: implications for the use of this test in the diagnostic approach of venous thromboembolism. Thrombosis Research, 69, 125-130.

Remy-Jardin, M., Remy, J., Wattinne, L. \& Giraud, F. (1992) Central pulmonary thromboembolism: diagnosis with spiral volumetric CT with the single-breath-hold technique: comparison with pulmonary angiography. Radiology, 185, 381-387.

Sackett, D.L., Haynes, R.B. \& Tugwell, P. (eds) (1985) Clinical Epidemiology: a basic science for clinical medicine, pp. 47-57. Little, Brown and Company, Boston.

Stein, P.D., Athanasoulis, C., Alavi, A., Greenspan, R.H., Hales, C.A., Saltzman, H.A., Vreim, C.E., Terrin, M.L. \& Weg, J.G. (1992) Complications and validity of pulmonary angiography in acute pulmonary embolism. Circulation, 85, 462-468.

Stein, P.D., Henry, J.W. \& Gottschalk, A. (1993a)The addition of clinical assessment to stratification according to prior cardiopulmonary disease further optimizes the interpretation of ventilation-perfusion lung scans in pulmonary embolism. Chest, 104, 1472-1476.

Stein, P.D., Hull, R.D., Salzman, H.A. \& Pineo, G. (1993b) Strategy for diagnosis of patients with suspected acute pulmonary embolism. Chest, 103, 1553-1559.

Teigen, C.L., Maus, T.P., Sheedy, P.F., II, Johnson, C.M., Stanson, A.W. \& Welch, T.J. (1993) Pulmonary embolism: diagnosis with electron-beam CT. Radiology, 188, 839-845.

Van Beek, E.J.R., Büller, H.R., Van Everdingen, J.J.E., Zwijnenburg, A. \& ten Cate, J.W. (1991) Diagnostic management of suspected pulmonary embolism in the Netherlands: results from a questionnaire. (Abstract). Thrombosis and Haemostasis, 65, 1171.

Van Beek, E.J.R., van den Ende, B., Berckmans, R.J., van der Heide, Y.T., Brandjes, D.P.M., Sturk, A. \& ten Cate, J.W. (1993) A comparative analysis of D-dimer assays in patients with clinically suspected pulmonary embolism. Thrombosis and Haemostasis, 70, 408-413.

Van der Meer, F.J.M., Rosendaal, F.R., Vandenbroucke, J.P. \& Briët, E. (1993) Bleeding complications in oral anticoagulant therapy: an analysis of risk factors. Archives of Internal Medicine, 153, 15571562 . 